\title{
Assessment of mitral regurgitation
}

\author{
T Irvine, X K Li, D J Sahn, A Kenny
}

Heart 2002;88(Suppl IV):iv1 1-iv19

M itral regurgitation (MR) is the most commonly encountered valve lesion in modern clinical practice. ${ }^{1}$ The range of pathologies producing regurgitant mitral valve dysfunction is broad (table 1 ) and the condition may be met in virtually any medical speciality. As echocardiography is the most widely available cardiac imaging modality, it is the technique which is routinely used to assess patients with suspected or known MR. While echo-Doppler is an excellent technique for detecting the presence of MR and defining the underlying pathological cause, assessing and/or quantifying the severity of the leak by echocardiography can at times be difficult. This reflects the fact that regurgitant flow through the mitral valve is a complex and dynamically changing process which may be impossible to characterise fully using a two dimensional imaging modality. Nevertheless, if MR is discovered on an echocardiographic examination it is extremely important to make an assessment of severity as this will be required to guide the patient's subsequent management.

What therefore is the optimal way to assess the severity of MR? Do potentially cumbersome quantitative echo-Doppler methods for calculation of regurgitant orifice area, regurgitant volume or regurgitant fraction add anything to a subjective assessment of severity (mild, moderate, severe) carried out by an experienced sonographer? Should already busy and overburdened echocardiography staff be stretching themselves further to perform complex quantitative techniques on MR patients? The answer is probably that detailed quantification is not necessary in the majority of patients. Noncardiologists need to know whether the regurgitation is significant enough to warrant further cardiological assessment; this does not require detailed quantitative information. Cardiologists use echo-Doppler grading of regurgitation severity in conjunction with patient symptoms and signs and occasionally invasive haemodynamic information to make decisions on the need for and timing of mitral valve surgery. In the majority of cases, quantitative echo-Doppler data are not required for this purpose and in fact current American College of Cardiology/American Heart Association guidelines for the management of $\mathrm{MR}^{2}$ do not make reference to any formal quantitative information. From time to time, however, situations will be encountered where there is discrepancy between the echocardiographic grading of regurgitation and the findings on clinical examination or other imaging modalities. In these circumstances more detailed quantitative echo-Doppler data may be required and it is important that the echocardiographer is conversant with the techniques involved. In addition, valve repair is increasingly performed on asymptomatic MR patients, and it may be that quantitative data such as regurgitant orifice area will become useful in the serial follow up of this group and in the decision of timing of surgery.

\section{DIAGNOSIS OF MITRAL REGURGITATION}

Echo-Doppler is an extremely sensitive tool for the detection of MR. Indeed, trivial or mild MR can be seen in $20-40 \%$ of subjects with structurally normal hearts ${ }^{3-5}$ and is of no clinical significance.

If significant MR is detected it is important not only to make an assessment of severity but also to make a judgement on the underlying cause. Careful inspection of the leaflet and subvalvar anatomy, along with the morphology and function of the left ventricle and papillary muscles, is required. From the list of conditions in table 1 it is evident that MR may be caused by either primary valvar pathology such as congenital heart disease, rheumatic heart disease or myxomatous degeneration, or secondary to miscellaneous pathologies such as mitral annular dilatation and/or left ventricular regional wall dysfunction (so-called "functional MR"). Defining the cause of the regurgitation is extremely important to guide further management of the patient. Primary valve pathologies will generally require valve repair or replacement when symptoms and/or haemodynamics dictate, whereas functional MR may respond to medical treatment of the underlying ventricular pathology, mitral annuloplasty or possibly revascularisation in the case of ischaemic left ventricular dysfunction.

Abbreviations: EROA, effective regurgitant orifice area; FC, flow convergence; LVOT, left ventricular outflow tract; $M R$, mitral regurgitation; TOE, transoesophageal echocardiography; TTE, transthoracic echocardiography; VC, vena contracta; VTI, velocity time integral

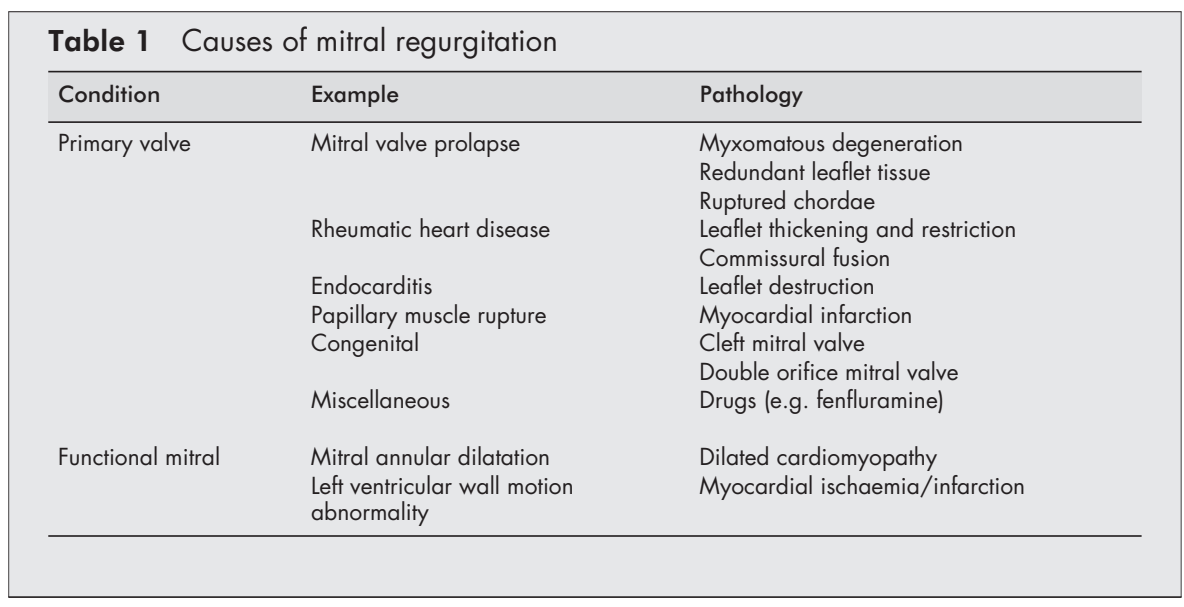




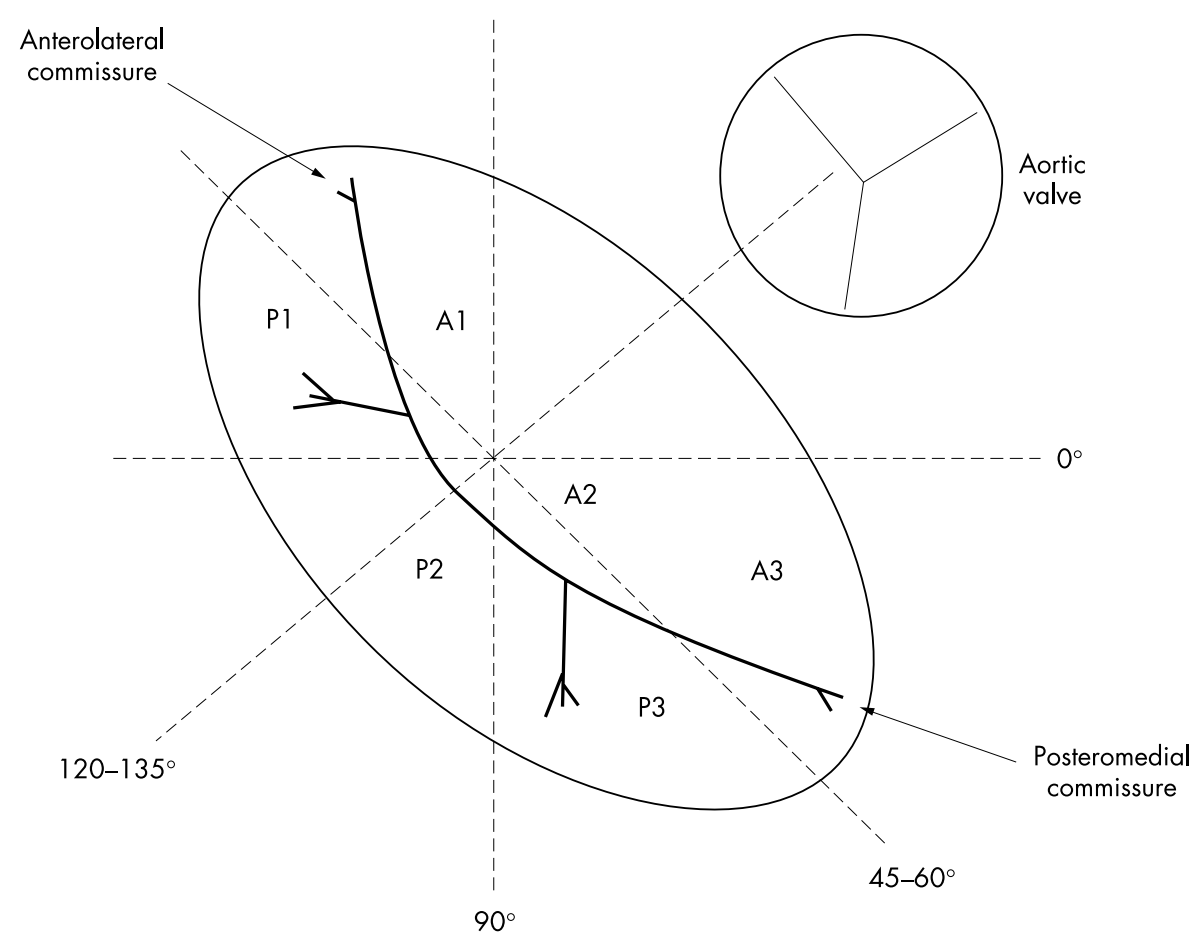

Figure 1 Diagram illustrating the subdivisions of the mitral valve leaflets. Planes indicate conventional multi-plane TOE views.

\author{
A1-A3 Subdivisions of anterior leaflet \\ P1-P3 Subdivisions of posterior leaflet \\ P1, P2 and P3 also referred to as anterolateral, central and posteromedial scallops of the posterior \\ leaflet respectively
}

Before discussing the pathologies affecting the mitral valve it is necessary to review the normal structure and function of the valve.

\section{NORMAL MITRAL VALVE STRUCTURE AND FUNCTION}

The normal mitral valve is a complex structure that relies on a precise interaction of all its components for normal function. The valve comprises the anterior and posterior leaflets, the subvalvar apparatus (chordae tendineae and papillary muscles), and the fibrous annulus. Failure of the normal function of any of these components may lead to regurgitation. Normal function of the regional walls of the left ventricle into which the papillary muscles insert is also required for valve competency. Figure 1 shows the structure of the mitral leaflets viewed from their superior aspect. The anterior mitral leaflet is a relatively long, semicircular structure. While the posterior leaflet is comparatively short in its leaflet edge to annulus dimension, it has a more extensive area of insertion to the annulus than the anterior leaflet. This fact is not readily appreciable on two dimensional echocardiographic imaging, where longitudinal cuts through the valve demonstrate leaflet width rather than area. For descriptive purposes discussed further below, both leaflets are subdivided into three components (fig l).

The chordae tendineae arise from the tips of the anterolateral and posteromedial papillary muscles and run to the free edges and body of the valve leaflets. Chords from the posteromedial muscle supply the medial half of both anterior and posterior leaflets, while those from the anterolateral muscle supply their lateral halves. As the chords are fixed in length, it is vital that the correct relationship is maintained between the leaflet edges and the papillary muscles to ensure that normal coaptation occurs. Any pathology affecting the structure and function of the papillary muscles or indeed their adjacent left ventricular walls may therefore produce valve dysfunction.

\section{PRIMARY MITRAL VALVE PATHOLOGY Rheumatic valve disease}

The echocardiographic features of rheumatic MR are generally easy to recognise. Thickening and calcification of the leaflet tissue and subvalvar apparatus with restricted leaflet motion are typical findings. Commissural fusion is commonly seen and there is usually a combination of mitral stenosis and regurgitation to varying degrees. A further clue may be involvement of other valves, most commonly the aortic.

\section{Mitral valve leaflet prolapse}

Mitral prolapse is most commonly caused by myxomatous degeneration of the valve leaflets and chordae tendineae. The leaflets become thickened and large amounts of redundant tissue may be present. Chordal elongation and rupture prevent normal apposition of the leaflet tips, and may produce valvar regurgitation of varying degrees. Less commonly, prolapse may result from direct damage to the papillary muscles or to the chordae through infarction or infection.

The echocardiographic diagnosis of mitral valve prolapse has historically been a contentious issue with a variety of definitions put forward. This lack of consensus has led to a wide range of reported prevalence of the condition (5-15\%). ${ }^{67}$ More recently, echocardiographic diagnostic criteria have been refined in the light of enhanced understanding of the three dimensional shape and motion of the mitral annulus. ${ }^{8-10}$ This has led to the introduction of the concept of "classic" mitral valve prolapse. Classic prolapse is defined as a maximum superior displacement of one or both mitral leaflets 
of $\geqslant 2 \mathrm{~mm}$ relative to the line connecting the annular hinge points during systole, and a maximum leaflet thickness of $\geqslant 5 \mathrm{~mm}$. In addition, "non-classic" prolapse is also described, with maximum displacement of $\geqslant 2 \mathrm{~mm}$ but a leaflet thickness $<5 \mathrm{~mm}$. Subjects with classic prolapse are at higher risk of complications than those with the non-classic form. ${ }^{11}$ Using these criteria, Freed and colleagues studied the transthoracic echocardiograms of 3500 subjects of the offspring cohort of the Framingham heart study. They showed a prevalence of $2.4 \%$ of mitral prolapse with approximately equal numbers of classic and non-classic forms. ${ }^{12}$

Of note is the fact that these criteria do not include an assessment of displacement of the coaptation point of the valve leaflets relative to the line connecting the annular hinge points. While previously proposed as an indicator of prolapse, posterior displacement of the coaptation point is not seen in the majority of subjects with classic and non-classic bileaflet prolapse. ${ }^{13}$ It is not therefore included in these most recent diagnostic criteria.

Prolapse may involve the entire leaflet or only a portion thereof. It is important to accurately define the extent of the leaflet involved in the prolapse, as this has implications for subsequent surgical management. While it may be possible to estimate the extent of involvement by transthoracic imaging, it is generally accepted that transoesophageal echocardiography is superior for this purpose ${ }^{14}$ (fig 1 ).

\section{FUNCTIONAL MITRAL REGURGITATION}

Mitral regurgitation may occur secondary to pathology outwith the mitral leaflets. ${ }^{15}{ }^{16}$ Dilatation of the left ventricle will lead to a stretch of the mitral valve annulus. If the annulus dilates to the point where complete leaflet coaptation is not possible, a leak will result usually producing a central jet of MR. A wall motion abnormality affecting the anterolateral or posteromedial left ventricular walls with or without direct papillary muscle involvement may also have an effect on mitral valve competency. Normal functioning of the valve relies on normal contraction of both the papillary muscles and the myocardium at their areas of insertion. Many authors describe the entity of papillary muscle dysfunction, but it is extremely unusual to see chronic, isolated dysfunction of these structures without involvement of the neighbouring myocardium. Normal myocardial contraction reduces the dimensions of both the short and long axes of the left ventricle and ensures that the correct relation between papillary muscles and leaflets is maintained during systole. An abnormality of systolic function of the regional left ventricular wall adjacent to a papillary muscle caused by ischaemia or infarction will impede this motion. The tip of the leaflet supplied by the papillary muscle will not be able to move back to its correct coaptation point and regurgitation will occur in an eccentric fashion behind the restricted leaflet. Dilatation of the left ventricle will produce displacement and restriction of motion of both papillary muscles which will add to the effects of annular dilatation in producing valvar regurgitation.

In some patients with functional MR secondary to ischaemic heart disease, the degree of regurgitation may increase during exercise since the resulting ischaemia will produce further deterioration in regional or global ventricular function. This "dynamic" regurgitation must be considered when assessing such patients.

\section{QUANTIFICATION OF MITRAL REGURGITATION Grading of severity}

As indicated above, in the majority of cases of MR a semi-quantitative estimate of the severity is sufficient for clinical purposes. It is customary to grade the lesion on a spectrum between mild and severe. A grading of severe indicates a large regurgitant volume, implying that the lesion is of major haemodynamic significance and may require surgical correction. A pragmatist might even argue that the only question to be answered is whether the regurgitation is severe or non-severe, since recommendations for surgical intervention are limited to the former group. Generally, however, an experienced sonographer will use several parameters to make an assessment of mild, moderate or severe regurgitation. Since this is a subjective assessment there will be a degree of variability between individual observers and institutions. Interobserver variability can, however, be minimised within individual institutions by the development of clear grading guidelines using the principles outlined below. It is advisable to keep the grading system as simple as possible, and there is little to be gained by broadening the range to include "mild to moderate" or "moderately severe".

\section{Colour Doppler regurgitant jet dimensions}

Colour Doppler regurgitant jet length and/or area has been used by a number of investigators to provide a semiquantitative measure of MR severity. To do so requires an assumption that the size of the jet is proportional to the regurgitant volume passing through the valve. It should be remembered, however, that the colour jet is a velocity map whose extent reflects the velocity of regurgitant flow at any moment rather than the absolute regurgitant volume at that point in time. It cannot therefore be regarded as the direct ultrasonic equivalent of a radiographic contrast angiogram. Despite this observation, the relation between colour jet dimensions and regurgitation severity has been demonstrated in several studies, ${ }^{17-19}$ but it must be pointed out that those studies demonstrating the best correlations related to "free" or unconstrained flows under strictly controlled flow conditions. Clinical studies indicate that the area of a free regurgitant jet averaged from multiple planes correlates well with angiographic grade of severity, and appears to be more robust than jet length alone. ${ }^{18}$ Correction of jet area for atrial area has also been studied and may improve accuracy. Helmcke ${ }^{18}$ demonstrated that jet areas of $<20 \%, 20-40 \%$, and $>40 \%$ of left atrial area predicted mild, moderate, and severe angiographic regurgitation, respectively. Such figures must be treated with caution, however, since atrial size is inherently linked to atrial pressure and compliance, both of which may themselves affect jet area (see below).

The relation between jet area and regurgitant volume becomes more tenuous when the effects of flow constraint by the atrial walls come into play. In the case of an eccentric jet of regurgitation, the jet will tend to travel across the floor and wall of the atrium rather than expanding freely into the centre of the chamber. Projected against the wall in this way, the eccentric jet cannot expand like a free jet and preferentially spreads outwards across the wall with a thin dimension perpendicular to the wall (the so-called Coanda effect). A number of studies have shown that these "wall jets" have smaller jet areas by colour flow mapping in conventional echocardiographic views than do free or unconstrained jets of the same regurgitant volume. ${ }^{20-22}$ This observation must be taken into account when using colour Doppler jet size to grade the severity of eccentric MR.

The size of the colour jet may also be influenced by any factor which affects the measured or displayed velocity of regurgitant flow (table 2). Such factors include instrument settings, transducer position, left atrial pressure and compliance, and left ventricular pressure and systolic function. ${ }^{23}$ Any or all of these may conspire to lead the unwary to the wrong conclusion regarding lesion severity. For example, high left ventricular pressures (caused by hypertension or co-existing aortic stenosis, for example) will produce high energy and hence high velocity jets of MR. Such jets will occupy a larger area than might be expected for any given degree of regurgitation, and this must be considered when reporting the severity of the lesion. A patient with acute, severe MR may display 
Table 2 Factors affecting colour Doppler regurgitant jet size

\begin{tabular}{|c|c|c|}
\hline Factor & & Relation to or effect on colour jet size \\
\hline \multicolumn{3}{|c|}{ Effects on measured or displayed flow velocities } \\
\hline \multirow[t]{4}{*}{ Haemodynamic effects } & LA compliance & Direct \\
\hline & LA pressure & Inverse \\
\hline & LV systolic function & Direct \\
\hline & LV pressure & Direct \\
\hline \multirow[t]{3}{*}{ Instrument settings } & Colour gain & Direct \\
\hline & Wall filter & Inverse \\
\hline & $\begin{array}{l}\text { Transducer frequency } \\
\text { Pulse repetition frequency }\end{array}$ & $\begin{array}{l}\text { Direct } \\
\text { Inverse }\end{array}$ \\
\hline \multicolumn{3}{|c|}{ Other factors affecting colour jet size } \\
\hline \multirow[t]{2}{*}{ Transducer position } & Relative to direction of flow & Doppler angle effects \\
\hline & Inadequate alignment to jet & Underestimation of true jet size \\
\hline Jet eccentricity & Coanda effect (see text) & Reduced jet dimensions \\
\hline
\end{tabular}

Table 3 Quantifiable entities for the assessment of mitral regurgitation

\begin{tabular}{lll}
\hline Quantifiable entity & Definition & Method of quantification \\
\hline Regurgitant volume $(\mathrm{ml})$ & $\begin{array}{l}\text { Volume regurgitating through } \mathrm{MV} \text { in } \\
\text { one systole }\end{array}$ & $\begin{array}{l}\text { Regurgitant volume as } \% \text { of total } \mathrm{MV} \text { [Regurgitant volume }(\mathrm{ml}) \div \mathrm{mitral} \text { inflow volume }(\mathrm{ml})] \times 100 \% \\
\text { Regurgitant fraction }(\%)\end{array}$ \\
EROA $\left(\mathrm{cm}^{2}\right)$ (quantitative Doppler) & $\begin{array}{l}\text { Effective area available for } \\
\text { regurgitant flow (mean over one }\end{array}$ & Regurgitant volume $(\mathrm{ml}) \div$ regurgitant orific VTI $(\mathrm{cm})$ \\
& $\begin{array}{l}\text { systole) } \\
\text { Effective area available for } \\
\text { regurgitant flow (instantaneous) }\end{array}$ & Regurgitant orifice flow rate $(\mathrm{ml} / \mathrm{s}) \div$ regurgitant orifice flow velocity $(\mathrm{cm} / \mathrm{s})$ \\
\hline
\end{tabular}

EROA, effective regurgitant orifice area; FC, flow convergence; LVOT, left ventricular outflow tract; MV, mitral valve; VTI, velocity time integral of mitral regurgitation continuous wave Doppler signal.

a relatively small area jet on colour flow mapping since in this situation left atrial pressures are high because of limited compliance of the receiving chamber.

Clearly therefore the use of colour jet dimensions to quantify MR must be undertaken with caution. In certain situations there is no doubt about the severity grade-a broad jet extending all the way back to the rear of the atrium is strongly suggestive of a severe leak. In other cases, especially in the case of eccentric jets, the situation may be less clear and several other parameters may be used to help determine severity. Most experienced sonographers will make an "eyeball" assessment of the size and extent of the regurgitant jet in a number of views. Formal measurement of jet area is generally not required. They will then give an indication of severity taking into account the above caveats. If doubt regarding severity remains, certain other qualitative parameters are available as adjuncts to aid in the assessment.

\section{Spectral Doppler}

The continuous wave Doppler envelope of the MR signal can provide clues to lesion severity. ${ }^{24}$ As the intensity of the Doppler signal is proportionate to the number of scatterers (that is, red blood cells) in the beam, severe lesions with large regurgitant volumes will generally produce high intensity Doppler envelopes. Conversely, the envelope in mild regurgitation will be fainter. Clearly, adequate beam alignment with the regurgitant flow is vital to make such observations valid.

\section{Pulmonary vein flow}

Examination of pulmonary vein flow by pulsed wave Doppler can be useful in the assessment of MR severity. ${ }^{25}{ }^{26}$ Rising left atrial pressure caused by significant regurgitation can cause a decrease in the normal forward flow through the pulmonary veins in late systole. Complete reversal of systolic flow may occur in severe MR, and is a useful indicator of its presence.
Depending on the direction of the regurgitant jet, flow reversal may be seen in a single pulmonary vein, in the two left or the two right sided veins, or in all four veins.

While pulmonary vein flow may be imaged by transthoracic echocardiography, transoesophageal imaging allows consistent measurement of flow velocities in all four pulmonary veins and is the imaging modality of choice for this purpose.

\section{Formal quantitative assessment}

On occasion there may still be uncertainty regarding lesion severity. In these situations a formal quantitative assessment may be useful. Three entities may be quantified-regurgitant volume, regurgitant fraction, and effective regurgitant orifice area (EROA). Table 3 lists the definitions and method of quantification of all three. Any or all of these parameters may be quantified, but we recommend the use of EROA as the concept of orifice area is one which is familiar to cardiac sonographers. Regurgitant volume may be a useful measure for serial follow up in an individual patient, but the absolute volume per se is unlikely to be a reliable guide to severity as it is dependent on haemodynamic variables and chamber size. Expressing the regurgitant volume as a ratio of total mitral inflow volume is a more standardised measure. Table 4 indicates the range of regurgitant fraction and EROA corresponding to conventional angiographic grades of regurgitation. Quantification of these parameters may be performed in two ways.

\section{Quantitative Doppler}

This technique allows calculation of volumetric flow at various points within the heart. For example, table 3 indicates that if mitral inflow and left ventricular outflow volumes can be calculated, then mitral regurgitant volume and fraction may be computed. If the velocity time integral of the regurgitant flow is also measured, EROA may be calculated. This is analogous to calculation of aortic valve area by the continuity equation which is itself an implementation of quantitative Doppler. 
Table 4 Relation between various quantitative parameters and severity of mitral regurgitation

\begin{tabular}{lllll}
\hline MR severity & Angiographic grade & $\begin{array}{l}\text { Regurgitant fraction } \\
(\%)\end{array}$ & EROA $\left(\mathrm{mm}^{2}\right)$ & VC width* $(\mathrm{cm})$ \\
\hline Mild & 1 & $<30$ & $<20$ & $<0.3$ \\
Moderate & 2 and 3 & $30-50$ & $20-40$ & $0.3-0.5$ \\
Severe & 4 & $>50$ & $>40$ & $>0.5$ \\
\hline
\end{tabular}

* Maximum width measurable from multiple views.

$E R O A$, effective regurgitant orifice area; $M R$, mitral regurgitation; VC, vena contracta

Sources: Dujardin et al, ${ }^{29}$ Hall et al. ${ }^{37}$

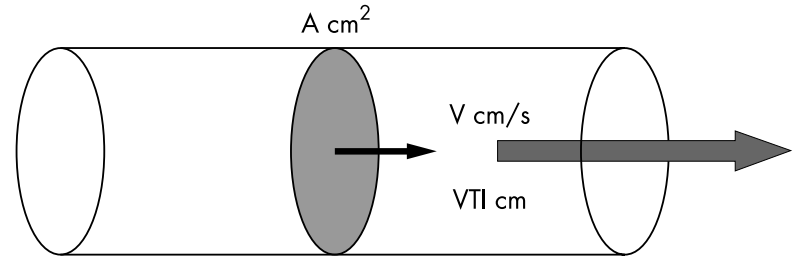

Instantaneous flow rate $=$ surface area $\times$ velocity $=A \times V \mathrm{ml} / \mathrm{s}$ Stroke volume $=\mathrm{A} \times \mathrm{VTI} \mathrm{ml}$

Figure 2 Principle of volume flow calculations for quantitative Doppler techniques

A detailed description of quantitative Doppler methodology has recently been published by the American Society of Echocardiography. ${ }^{27}$ The technique is based on the principle that volume flow rate is equal to the product of the cross sectional surface area available for flow at any point in a flow system and the velocity of flow at that surface. Depending on the

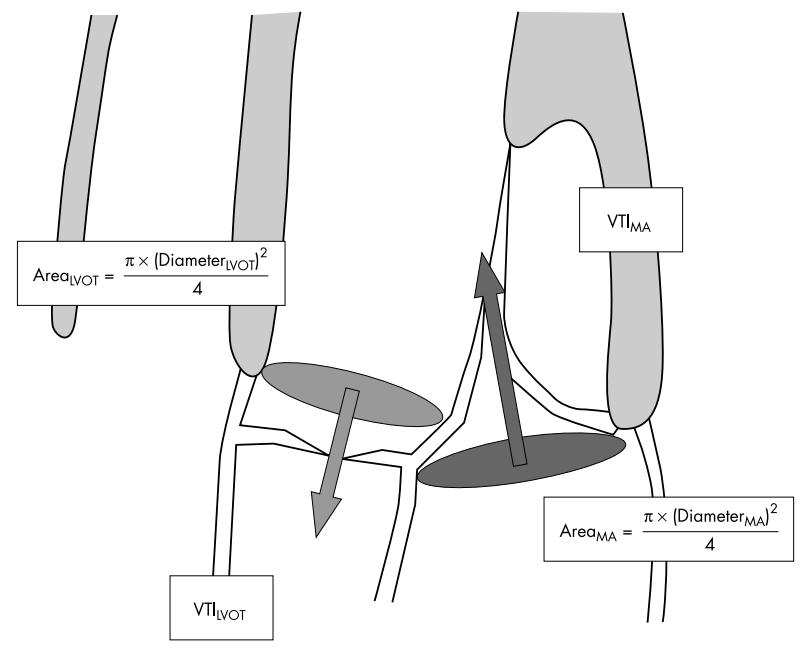

Mitral inflow volume $=$ Area $_{M A} \times \mathrm{VTI}_{\mathrm{MA}}$

Left ventricular outflow volume $=$ Area $_{\mathrm{LVOT}} \times \mathrm{VTI}_{\mathrm{LVOT}}$

Regurgitated volume $=\left(\right.$ Area $\left._{M A} \times \mathrm{VTI}_{\mathrm{MA}}\right)-\left(\right.$ Area $\left._{\mathrm{LVOT}} \times \mathrm{VTI}_{\mathrm{LVOT}}\right)$

$$
\mathrm{EROA}^{*}=\frac{\text { Regurgitated volume }}{\mathrm{VTI} \text { mitral regurgitation by CW Doppler }}
$$

Figure 3 Calculation of regurgitant volume and effective regurgitant orifice area (EROA) by quantitative Doppler. *EROA by this method gives the mean value for one cycle. LVOT, left ventricular outflow tract; MA, mitral annulus. parameters measured, instantaneous volume flow rate or stroke volume (that is, the volume passing through the selected surface in one cardiac cycle) can be calculated as shown in fig 2.

This principle may be applied to the mitral annulus and the left ventricular outflow tract (LVOT) to calculate mitral valve diastolic inflow and LVOT systolic outflow, respectively, and hence mitral regurgitant volume and EROA (fig 3). Mitral annular area is calculated by measuring the diameter of the mitral annulus in the apical 4 chamber view during diastole and assuming circular cross section. Although the mitral annulus is not perfectly circular, it has been shown that an assumption of circular geometry yields satisfactory results in this context. Mitral annular velocity time integral (VTI) is measured by placing the PW Doppler sample volume at the level of the annulus as shown. Clearly it must be assumed that the velocity data measured at that point reflect the velocities across the entire annular plane (that is, that the flow profile is flat), but it also appears that this assumption yields satisfactory accuracy. The area of the LVOT is calculated by measuring its diameter in the parasternal long axis view and assuming circular geometry. VTI data are collected in the apical five chamber view with the PW sample volume placed at the same level in the LVOT as the previous diameter measurement. Finally, the VTI of the mitral regurgitant signal itself must also be recorded by CW Doppler from the apical 4 chamber view.

Quantitative Doppler calculations are therefore time consuming and require skill and expertise for their implementation. Incorrect diameter measurements will produce large errors since the value must be squared to generate the cross sectional area. Several measurements of each parameter (B mode and Doppler) should be made and averaged to minimise error. In addition the calculation will not be valid in the presence of significant aortic regurgitation. In experienced hands, however, quantitative Doppler provides data of sufficient accuracy for clinical use and is generally regarded as the gold standard echo Doppler technique for quantification of valvar regurgitation. ${ }^{28} 29$

\section{Flow convergence method}

The flow convergence (FC) method provides an alternative to quantitative Doppler for the calculation of EROA. It refers to the organised area of flow just proximal to the regurgitant orifice. ${ }^{30}$ This is a more attractive option for flow quantification than the turbulent and disorganised regurgitant jet. The FC principle is best understood by considering the theoretical example of a flow field approaching a point orifice on a flat plate. As flow converges on the orifice, it accelerates along a series of multidirectional streamlines (fig 4 ) to reach maximum velocity at the orifice itself. If colour Doppler is used to image the flow field, it will be found that the velocity of flow will exceed the first colour Doppler aliasing velocity $(v \mathrm{~cm} / \mathrm{s})$ at a certain distance $r \mathrm{~cm}$ from the orifice. At this point an abrupt colour change will occur. In the case of a point orifice, each flow streamline will exceed the aliasing velocity at the same distance $(r \mathrm{~cm})$ from the orifice as shown. Two dimensional colour Doppler imaging of the flow field will therefore show an arc of abrupt colour change of 


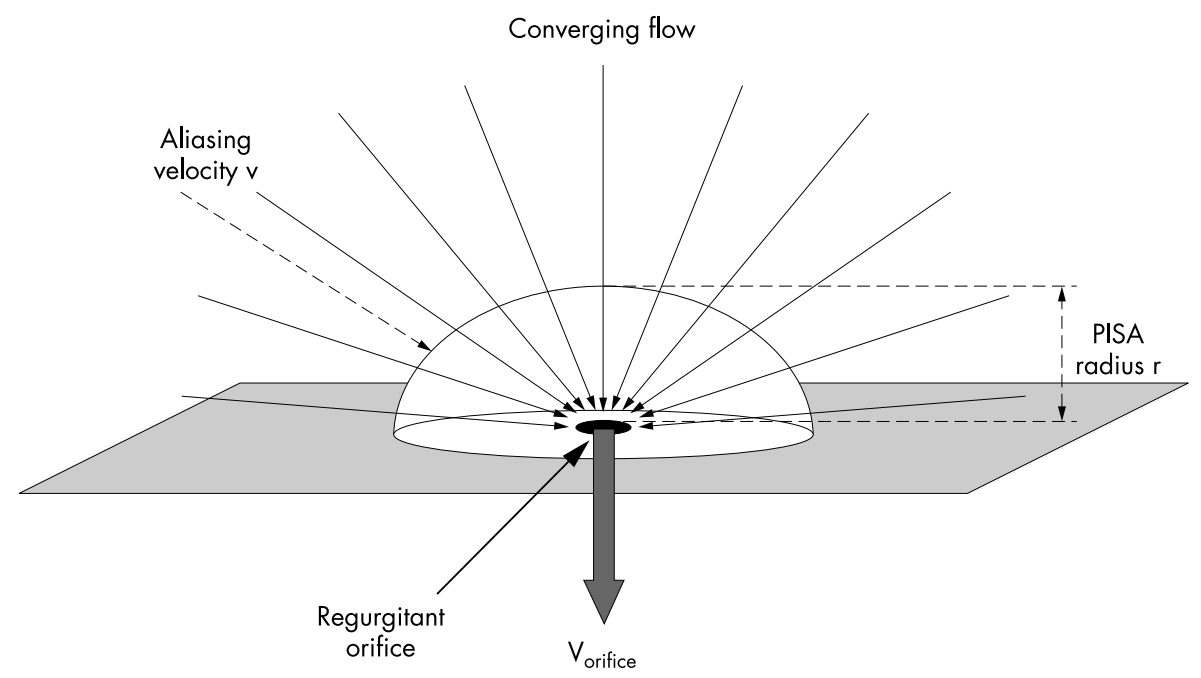

Figure 4 Schematic representing the formation of the flow convergence (FC) flow zone.

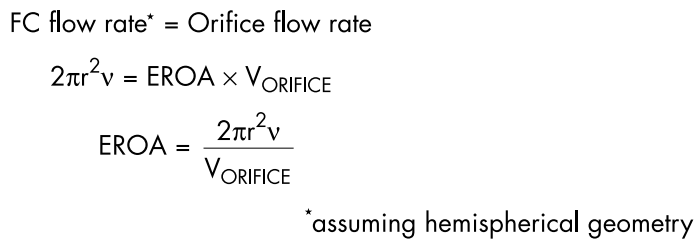

radius $r \mathrm{~cm}$ overlying the orifice. As the flow continues to accelerate towards the orifice, further arcs of progressively smaller radii will appear each time the velocity exceeds a multiple of the aliasing threshold.

Since flow approaches the orifice from all directions, in three dimensional space each arc in our point orifice model will appear as a hemisphere of radius $r \mathrm{~cm}$ (fig 4). This "structure" is known as the region of flow convergence, and its physical area can be calculated as $2 \pi r^{2}$ (surface area of a hemisphere). Although in clinical practice the regurgitant orifice will be of finite size and unpredictable shape, the FC phenomenon is still encountered. Figure 5 shows a transoesophageal example of a large FC produced by severe MR secondary to posterior leaflet prolapse.

Since volume flow rate may be calculated as the product of surface area of flow and the flow velocity, the instantaneous volume flow rate at the FC will be $2 \pi r^{2} v$. By the same principle, the instantaneous flow rate at the regurgitant orifice (regurgitant flow rate) will equal the product of the EROA and the velocity of flow through the orifice (by CW Doppler) at that instant. By the principle of conservation of mass, the volume flow rate at the FC must equal the regurgitant flow rate. Using the continuity equation, the EROA may be calculated as follows:

Orifice flow rate $=$ flow convergence flow rate

$$
\begin{gathered}
\therefore E R O A \times V_{\text {orifice }}=2 \pi r^{2} v \\
\therefore E R O A=\frac{2 \pi r^{2} v}{V_{\text {orifice }}}
\end{gathered}
$$

where $\mathrm{r}=\mathrm{FC}$ radius, $\mathrm{v}=$ colour Doppler aliasing velocity, and $\mathrm{V}_{\text {orifice }}=$ velocity at regurgitant orifice by continuous wave Doppler.

Although based on a theoretical model, a number of clinical studies have shown that these FC based flow and EROA computations produce results comparable to quantitative Doppler techniques. ${ }^{31-33}$ The assumption of hemispherical geometry of the FC is central to the calculation, and it appears that the first

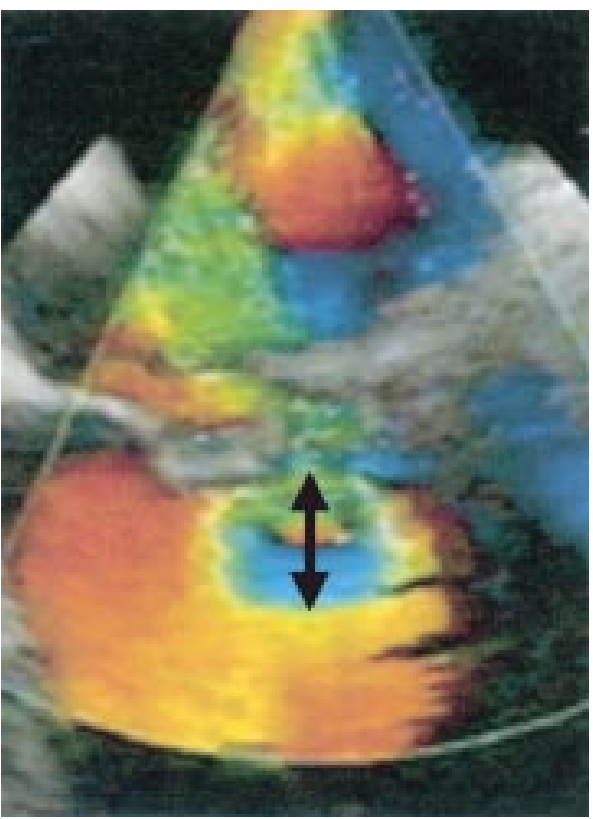

Figure 5 Example of the flow convergence $(\mathrm{FC})$ phenomenon on transoesophageal imaging of posterior mitral leaflet prolapse with severe regurgitation (arrow indicates FC radius measurement).

(outer) aliasing boundary conforms more closely to this shape than successive, smaller FC zones. It is recommended therefore that the radius of the outermost FC (fig 5) is measured and used for flow computations.

It is important to realise that the EROA computed in this way reflects the area at that instant in time since the calculation is based on flow velocities which change dynamically over the cardiac cycle. In clinical practice, the operator will freeze the 2D colour Doppler image and scroll back through the digital cineloop until the frame where the FC size is maximal is found. This is assumed to correspond to the peak regurgitant 
flow rate since the velocity of flow will be highest at that point in time and the FC aliasing boundary will be at its furthest distance from the valve. FC flow rate is calculated from this frame using the above methodology. CW Doppler is then applied through the regurgitant orifice and the peak velocity of regurgitant flow recorded. In this way it can be assumed that FC and orifice flow rates correspond to the same point in time. The EROA thus generated will also coincide with the peak regurgitant flow rate. Quantitative Doppler on the other hand generates a mean EROA as its calculations are based on the regurgitant stroke volume and the VTI of the regurgitant flow signal. In practice, however, while there is minor variability in the EROA over the cardiac cycle, the difference between the peak and mean EROA is negligible for clinical purposes.

Thomas and colleagues have shown that the FC method can be simplified as follows, without significant loss of accuracy. ${ }^{34}$ If it is assumed that the peak pressure difference from the left ventricle to left atrium in systole is in the order of $100 \mathrm{~mm} \mathrm{Hg}$ (a reasonable assumption in the presence of normal left ventricular function), a mitral regurgitant jet of $5 \mathrm{~m} / \mathrm{s}$ peak velocity will result. If the colour Doppler aliasing velocity is set to $40 \mathrm{~cm} / \mathrm{s}$, the $\mathrm{FC}$ calculation simplifies to EROA $=\mathrm{r}^{2} / 2$. The amount of time saved by using this abbreviated version is debatable, however, since the majority of commercially available echocardiographic systems have on-board computation packages which greatly facilitate the formal FC calculations.

While the hemispherical assumption appears to work well for "free" or unconstrained FCs, in cases where flow proximal to a regurgitant orifice is constrained (for example, by an adjacent wall in the case of eccentric regurgitation) the FC shape may not be a true hemisphere. In such cases it is reasonable to adjust the formula by measuring the angle $\alpha$ subtended by the arc of the constrained FC and correcting the EROA calculation by a factor of $\alpha / 180 .^{35}$

A further potential difficulty with the FC methodology can be the identification of the regurgitant orifice when making the radius measurement to the first aliasing boundary. To overcome this problem, the concept of "inter-aliasing distance" has been introduced. ${ }^{36}$ This is based on the principle that by conservation of mass, the flow rate at any of the multiple FC zones approaching the orifice must be equal. A mathematical model has been developed which uses the distance between the first and second aliasing boundaries (the inter-aliasing distance) to generate a derived radius which may be used in FC flow computations. The mathematics are complex but the model does circumvent the need to define the position of the orifice.

\section{Vena contracta measurements}

The vena contracta (VC) is the narrowest portion or "neck" of the regurgitant jet which occurs at or just beyond the regurgitant orifice (figs 6 and 7). Although flow in this region is of high velocity, the flow pattern is organised into a series of parallel flow lines. Distal to the VC, flow becomes progressively more turbulent and disorganised as blood in the receiving chamber is entrained by the expanding regurgitant jet. Theoretically, the cross sectional area of the VC should bear a direct relation to the regurgitant orifice area. As the mitral regurgitant orifice in the clinical setting is likely to be complex and unpredictable in shape, to make such an area measurement would require the regurgitant jet to be imaged in a short axis plane. Hall and colleagues have shown that in the clinical setting, localisation of the narrowest portion of the regurgitant jet during short axis transthoracic imaging is difficult. ${ }^{37}$ They do, however, demonstrate a clinically useful correlation between the width of the VC measured in zoom mode during long axis imaging of the jet in the parasternal and apical views, and the EROA measured by quantitative Doppler. The relation between VC width and EROA has been

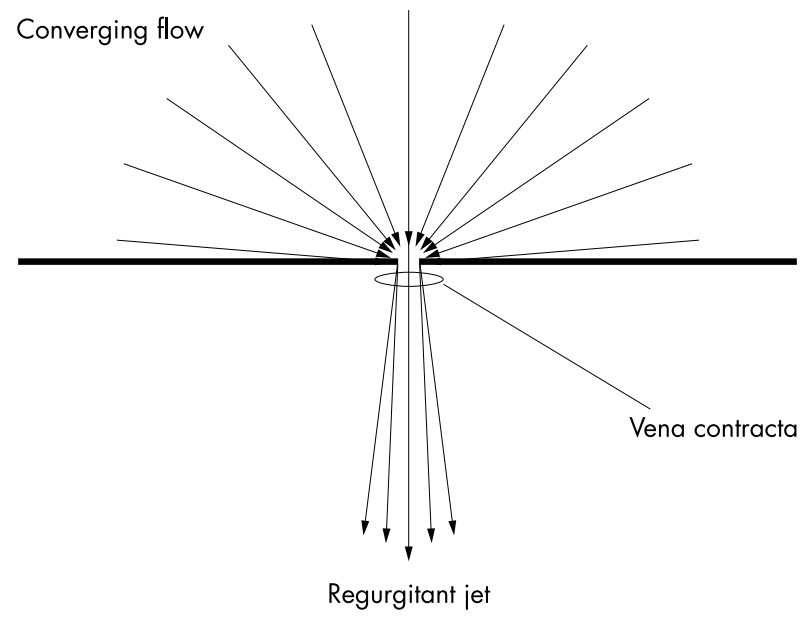

Figure 6 Schematic depicting the position of the vena contracta in a regurgitant flow field.

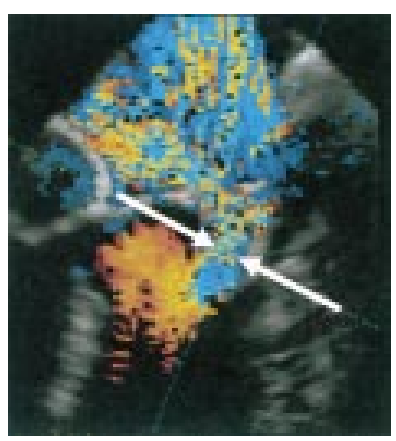

Figure 7 Transoesophageal example of the vena contracta (arrows) in a patient with severe, eccentric mitral regurgitation.

confirmed by other investigators, ${ }^{38-40}$ and appears to hold true even in eccentric MR. ${ }^{41}$

Clearly there are limitations to using a one dimensional measurement (VC width) for the quantification of a two dimensional structure (EROA), but Hall shows that the former is a useful tool for quantification of MR with VC widths of $<0.3 \mathrm{~cm}$ and $>0.5 \mathrm{~cm}$ indicating mild and severe regurgitation, respectively (table 4). There are limitations to the technique, however. Even in the long axis view, localisation of the VC is difficult and requires considerable operator skill and patience. In addition, since the regurgitant orifice is unlikely to be regular in shape and may occur at any point along the mitral valve closure line, the VC must be identified and measured in a number of planes and "off-axis" views. Finally, since the absolute dimensions of the VC are small (several millimetres) there are issues regarding the axial versus lateral resolution of the ultrasound system. Axial resolution is related to wavelength and pulse duration and is superior to lateral resolution which is dependent on scan line spacing and beam width. VC measurements made in the direction of the axial imaging plane (parasternal views in the case of MR) should therefore be more accurate than those made in the lateral plane (apical views for MR). This effect becomes more significant at greater imaging depths since scan lines are radially distributed and become more separated as distance from the probe increases. The issue of axial versus lateral resolution is likely to be less pronounced in newer ultrasound systems which scan multiple lines simultaneously, but it is still advisable to make VC measurements in the axial plane where possible.

TRANSTHORACIC VERSUS TRANSOESOPHAGEAL IMAGING

All of the quantitative techniques discussed above may be performed by both transthoracic (TTE) and transoesophageal 
(TOE) echocardiography. It is certainly not necessary to perform TOE imaging in all patients with MR, but the superior resolution of that modality makes it easier to visualise and measure the FC zone if formal quantification is required. TOE also allows localisation of the vena contracta and measurement of its dimensions. ${ }^{38} 40$

Doppler examination of pulmonary vein flow is also easier with TOE, although in our experience the introduction of ultrasonic contrast agents has made this possible in the majority of patients by TTE.

TOE imaging is extremely useful for the assessment of patients with mitral valve prolapse, particularly if surgical repair is contemplated. TOE is superior to TTE for the determination of the extent and distribution of leaflet involvement, both of which are of major importance in deciding on the suitability of the valve for repair.

It is advisable to use both TTE and TOE in the assessment of prosthetic mitral valve regurgitation, especially in the case of mechanical valves. This will minimise the effect of "shielding" by the metal components of the valve. For mitral valve prostheses, TOE imaging also allows differentiation between valvar and paravalvar regurgitation which may be difficult by transthoracic imaging. It also allows precise localisation of the site and extent of the paravalvar leak.

\section{OTHER CONSIDERATIONS}

In addition to quantifying the severity of regurgitation, it is important to assess the size and function of the left ventricle. In the early stages, the large volume overload associated with severe MR produces a left ventricle which is dilated with hyperdynamic function, where the end systolic cavity volume is small compared to the end diastolic volume. With time, however, sustained volume overload will ultimately lead to left ventricular dysfunction, and it is clearly desirable to correct severe MR before significant dysfunction has occurred. In this situation, end systolic chamber size is a more sensitive marker of impending ventricular dysfunction than end diastolic dimensions. The American Heart Association/American College of Cardiology recommend valve surgery in those patients with severe MR and a left ventricular end systolic diameter of $4.5 \mathrm{~cm}$ or greater even if the patient is asymptomatic. ${ }^{2}$ Regular monitoring of left ventricular dimensions in patients with significant MR is therefore of the utmost importance.

Pulmonary artery systolic pressure should also be monitored where possible. The onset of pulmonary hypertension is an ominous sign in the setting of MR, and its early detection is mandatory.

Care must be taken in the evaluation of those MR patients who are apparently symptomatic with significant limitation to their activity, but whose echocardiographic study indicates "non-severe" MR. In this situation it is useful to reproduce the symptoms by exercising the patient (for example, with two minutes of sit-ups or leg raising) and repeating the study immediately after. The altered haemodynamics on exercise may increase the degree of MR and it is possible to quantify this formally by calculating EROA pre- and post-exercise. In addition, in those patients with functional MR secondary to regional or global left ventricular ischaemia, the true extent of regurgitation will only be seen if ischaemia is induced either by exercise or pharmacological stress echocardiography.

Exercise echocardiography has also been used to assess the contractile reserve of the left ventricle in MR patients. The post-exercise end systolic volume has been shown to be an effective predictor of left ventricular function after mitral valve repair. $^{42}$

\section{FUTURE DIRECTIONS}

Quantitative Doppler is a cumbersome technique. A number of measurements must be made to calculate volume flow rates through the LVOT and mitral annulus to ultimately generate mitral regurgitant volumes. Error will arise if any one of these measurements is made incorrectly. More sophisticated echoDoppler methods are available which use spatio-temporal integration of digital flow velocities through these regions to compute volume flow rates automatically. One such implementation is automated cardiac output measurement (ACOM, Toshiba Imaging Systems). This method has been validated by a number of investigators and is a potential alternative to complex quantitative Doppler techniques. ${ }^{43}{ }^{44}$

Three dimensional echocardiography is a new technique which has been shown to be superior to conventional methods for the calculation of cardiac chamber volumes. Work is currently in progress examining its role in the quantification of regurgitant flow. A new three dimensional digital colour Doppler method has recently been introduced which should allow more accurate quantification of mitral and LVOT flow volumes, and hence regurgitant volumes. ${ }^{45}$ Three dimensional echocardiography also has potential advantages over two dimensional methods in that flow events such as the FC can be viewed in their entirety, obviating the need to make a hemispherical shape assumption for surface area computations. ${ }^{46}$ Since the entire regurgitant jet can be imaged and stored by three dimensional echocardiography, precise localisation of the VC should also be possible.

\section{SUMMARY}

Echo-Doppler is an extremely effective tool both for determining the pathology responsible for MR and for making an assessment of its severity. In the majority of patients, an "eyeball" grading of severity on a range from mild to severe is generally sufficient for clinical purposes. More formal quantitative approaches may be required in cases where there is discrepancy between this eyeball assessment and other clinical findings, and all cardiac sonographers should be conversant with at least one of these techniques. Exercise echocardiography may be required to assess the degree of regurgitation during the haemodynamic conditions occurring at the time of the patient's symptoms. Exercise or pharmacological stress echocardiography may be also required in those patients with MR related to dynamic myocardial ischaemia. Serial measurement of left ventricular dimensions and pulmonary artery pressure is vitally important to detect impending problems with left ventricular dysfunction or pulmonary hypertension. Finally, as mitral valve repair becomes a safer procedure contemplated for asymptomatic patients, serial monitoring of EROA may also become advantageous to aid the decision of timing of valve surgery.

\section{....................}

Authors' affiliations

T Irvine, A Kenny, Cardiothoracic Unit, Freeman Hospital, Newcastle upon Tyne, UK

X K Li, D J Sahn, Department of Pediatric Cardiology, Oregon Health and Science University, Portland, Oregon, USA

Correspondence to: Dr Antoinette Kenny, Cardiothoracic Unit, Freeman Hospital, High Heaton, Newcastle upon Tyne NE7 7DN, UK; antoinette.kenny@ncl.ac.uk

\section{REFERENCE}

1 Jones EC, Devereux RB, Roman M, et al. Prevalence and correlates of mitral regurgitation in a population-based sample (the strong heart study). Am J Cardiol 2001;87:298-304.

2 Bonow RO, Carabello BA, deleon AC, et al. ACC/AHA guidelines for the management of patients with valvular heart disease: executive summary. A report of the American College of Cardiology/American Heart Association task force on practice guidelines (committee on management of patients with valvular heart disease). Circulation 1998;98:1949-84.

3 Yoshida K, Yoshikawa J, Shakudo M, et al. Color Doppler evaluation of valvular regurgitation in normal subjects. Circulation 1988;78:840-7.

4 Choong CY, Abascal VM, Weyman J, et al. Prevalence of valvular regurgitation by Doppler echocardiography in patients with structurally normal hearts by two-dimensional echocardiography. Am Heart J 1989; 1 17:636-42. 
5 Biava G, Delfino M, Lagana B, et al. Prevalence of valvular regurgitation in structurally normal hearts: a colour-Doppler study. Coronary Artery Disease 1997;8:559-63

6 Markiewicz W, Stoner J, London E, et al. Mitral valve prolapse in one hundred presumably healthy young females. Circulation 1976:53:464-73.

7 Procacci PM, Savran SV, Schreiter SL, et al. Prevalence of clinical mitral valve prolapse in 1169 young women. N Engl J Med 1976;294:1086-8

8 Levine RA, Triulzi MO, Harrigan P, et al. The relationship of mitral annular shape to the diagnosis of mitral valve prolapse. Circulation 1987;75:756-67.

9 Levine RA, Stathogiannis E, Newell JB, et al. Reconsideration of echocardiographic standards for mitral valve prolapse: lack of association between leaflet displacement isolated to the apical four chamber view and independent echocardiographic evidence of abnormality [review]. J Am Coll Cardiol 1988;11:1010-19.

10 Levine RA, Handschumacher MD, Sanfilippo AJ, et al. Three-dimensional echocardiographic reconstruction of the mitral valve, with implications for the diagnosis of mitral valve prolapse. Circulation 1989:80:589-98.

11 Marks AR, Choong CY, Sanfilippo AJ, et al. Identification of high-risk and low-risk subgroups of patients with mitral-valve prolapse. N Engl J Med 1989;320:1031-6.

12 Freed LA, Levy D, Levine RA, et al. Prevalence and clinical outcome of mitral-valve prolapse. N Engl J Med 1999;341:1-7.

13 Weyman AE. Principles and practice of echocardiography. Philadelphia: Lippincott Williams \& Wilkins, 1994;446-56

14 Flachskampf FA, Decoodt P, Fraser AG, et al. Recommendations for performing transoesophageal echocardiography. Eur J Echocardiogr $2001 ; 2: 8-21$.

15 He S, Fontaine AA, Schwammenthal E, et al. Integrated mechanism for functional mitral regurgitation: leaflet restriction versus coapting force: in vitro studies. Circulation 1997;96:1826-34.

16 Yiu SF, Enriquez-Sarano M, Tribouilloy C, et al. Determinants of the degree of functional mitral regurgitation in patients with systolic left ventricular dysfunction: a quantitative clinical study. Circulation 2000;102:1400-6

17 Miyatake K, Izumi S, Okamoto M, et al. Semiquantitative grading of severity of mitral regurgitation by real-time two-dimensional Doppler flow imaging technique. J Am Coll Cardiol 1986:7:82-8.

18 Helmcke F, Nanda NC, Hsiung MC, et al. Color Doppler assessment of mitral regurgitation with orthogonal planes. Circulation 1987;75:175-83.

19 Spain MG, Smith MD, Grayburn PA, et al. Quantitative assessment of mitral regurgitation by Doppler color flow imaging: angiographic and hemodynamic correlations. J Am Coll Cardiol 1989;13:585-90.

20 Chen C, Thomas JD, Anconia J, et al. Impact of impinging wall jet on color Doppler quantification of mitral regurgitation. Circulation 1991;84:712-20.

21 Enriquez-Sarano M, Tajik AJ, Bailey KR, et al. Color flow imaging compared with quantitative Doppler assessment of severity of mitral regurgitation: influence of eccentricity of jet and mechanism of regurgitation [erratum appears in J Am Coll Cardiol 1993;22:342]. J Am Coll Cardiol 1993:21:1211-19.

22 Shiota $T$, Jones $M$, Teien $D$, et al. Color Doppler regurgitant jet area for evaluating eccentric mitral regurgitation: an animal study with quantified mitral regurgitation. J Am Coll Cardiol 1994;24:813-19.

23 Sahn DJ. Instrumentation and physical factors related to visualization of stenotic and regurgitant jets by Doppler color flow mapping. J Am Coll Cardiol 1988; 12:1354-65.

24 Utsunomiya T, Patel D, Doshi R, et al. Can signal intensity of the continuous wave Doppler regurgitant jet estimate severity of mitral regurgitation? Am Heart J 1992;123:166-71.

25 Kamp O, Huitink $H$, van Eenige $M$, et al. Value of pulmonary venous flow characteristics in the assessment of severity of native mitral valve regurgitation: an angiographic correlated study. J Am Soc Echocardiogr 1992;5:239-6

26 Teien DE, Jones $M$, Shiota $T$, et al. Doppler evaluation of severity of mitral regurgitation: relation to pulmonary venous blood flow patterns in an animal study. J Am Coll Cardiol 1995;25:264-8.
27 Quinones MA Otto CM Stoddard M, et al. Recommendations for quantification of Doppler echocardiography: a report from the Doppler quantification task force of the nomenclature and standards committee of the American Society of Echocardiography. J Am Soc Echocardiogr 2002; 15: 167-84.

28 Enriquez-Sarano M, Bailey KR, Seward JB, et al. Quantitative Doppler assessment of valvular regurgitation. Circulation 1993;87:841-8.

29 Dujardin KS, Enriquez-Sarano M, Bailey KR, et al. Grading of mitral regurgitation by quantitative Doppler echocardiography: calibration by left ventricular angiography in routine clinical practice. Circulation 1997;96:3409-15

30 Recusani F, Bargiggia GS, Yoganathan AP, et al. A new method for quantification of regurgitant flow rate using color Doppler flow imaging of the flow convergence region proximal to a discrete orifice. An in vitro study. Circulation 1991;83:594-604.

31 Rivera JM, Vandervoort PM, Thoreau DH, et al. Quantification of mitral regurgitation with the proximal flow convergence method: a clinical study. Am Heart J 1992;124:1289-96.

32 Vandervoort PM, Rivera JM, Mele D, et al. Application of color Doppler flow mapping to calculate effective regurgitant orifice area. An in vitro study and initial clinical observations. Circulation 1993;88:1150-6.

33 Rivera JM, Mele D, Vandervoort PM, et al. Effective regurgitant orifice area in tricuspid regurgitation: clinical implementation and follow-up study. Am Heart J 1994;128:927-33.

34 Pu M, Prior DL, Fan X, et al. Calculation of mitral regurgitant orifice area with use of a simplified proximal convergence method: initial clinical application. J Am Soc Echocardiogr 2001;14:180-5

35 Pu M, Vandervoort PM, Griffin BP, et al. Quantification of mitral regurgitation by the proximal convergence method using transesophageal echocardiography. Clinical validation of a geometric correction for proximal flow constraint. Circulation 1995;92:2169-77.

36 Sitges $M$, Jones $M$, Shiota $T$, et al. Interaliasing distance of the flow convergence surface for determining mitral regurgitant volume: a validation study in a chronic animal model. J Am Coll Cardiol 2001;38:1195-202.

37 Hall SA, Brickner ME, Willett DL, et al. Assessment of mitral regurgitation severity by Doppler color flow mapping of the vena contracta. Circulation 1997;95:636-42

38 Grayburn PA, Fehske W, Omran H, et al. Multiplane transesophageal echocardiographic assessment of mitral regurgitation by Doppler color flow mapping of the vena contracta. Am J Cardiol 1994;74:912-17.

39 Fehske W, Omran H, Manz M, et al. Color-coded Doppler imaging of the vena contracta as a basis for quantification of pure mitral regurgitation. Am J Cardiol 1994;73:268-74.

40 Heinle SK, Hall SA, Brickner ME, et al. Comparison of vena contracta width by multiplane transesophageal echocardiography with quantitative Doppler assessment of mitral regurgitation. Am J Cardiol 1998;81:175-9.

41 Zhou X, Jones $M$, Shiota T, et al. Vena contracta imaged by Doppler color flow mapping predicts the severity of eccentric mitral regurgitation better than color jet area: a chronic animal study. J Am Coll Cardiol 1997;30: 1393-8.

42 Leung DY, Griffin BP, Stewart WJ, et al. Left ventricular function after valve repair for chronic mitral regurgitation: predictive value of preoperative assessment of contractile reserve by exercise echocardiography. J Am Coll Cardiol 1996;28:1 198-205.

43 Hozumi T, Yoshida K, Akasaka T, et al. Automated assessment of mitra regurgitant volume and regurgitant fraction by a newly developed digita color Doppler velocity profile integration method. Am J Cardiol 1997:80:1325-30

44 Sun JP, Yang XS, Qin JX, et al. Quantification of mitral regurgitation by automated cardiac output measurement: experimental and clinical validation. J Am Coll Cardiol 1998;32:1074-82.

45 Rusk RA, Li XN, Mori Y, et al. Direct quantification of transmitral flow volume with dynamic 3-dimensional digital color Doppler: a validation study in an animal model. J Am Soc Echocardiogr 2002;15:55-62.

46 Shiota $T$, Jones $M$, Delabays $A$, et al. Direct measurement of three-dimensionally reconstructed flow convergence surface area and regurgitant flow in aortic regurgitation: in vitro and chronic animal mode studies. Circulation 1997;96:3687-95. 